\title{
Therapeutic effect of metformin in the treatment of endometrial cancer (Review)
}

\author{
NAN MU ${ }^{1 *}$, TINGTING XU ${ }^{1 *}$, MINGXIAO GAO ${ }^{2 *}$, MEI DONG $^{2}$, QING TANG $^{1}$, LI HAO $^{1}$, \\ GUIQING WANG $^{1}$, ZENGHUI LI ${ }^{1}$, WENSHUANG WANG ${ }^{1}$, YING YANG ${ }^{1}$ and JIANQING HOU ${ }^{1}$ \\ Departments of ${ }^{1}$ Gynecology and Obstetrics and ${ }^{2}$ Cardiology, Yantai Yuhuangding Hospital \\ Affiliated to Qingdao University, Yantai, Shandong 264000, P.R. China
}

Received March 2, 2020; Accepted July 14, 2020

DOI: $10.3892 / \mathrm{ol} .2020 .12017$

\begin{abstract}
The present review aims at reviewing the role of metformin in the treatment of endometrial cancer (EC). According to the literature, excessive estrogen levels and insulin resistance are established risk factors of EC. As a traditional insulin sensitizer and newly discovered anticancer agent, metformin directly and indirectly inhibits the development of EC. The direct mechanisms of metformin include inhibition of the LKB1-AMP-activated protein kinase-mTOR, PI3K-Akt and insulin-like growth factor 1-related signaling pathways, which reduces the proliferation and promotes the apoptosis of EC cells. In the indirect mechanism, metformin increases the insulin sensitivity of body tissues and decreases circulating insulin levels. Decreased levels of insulin increase the blood levels of sex hormone binding globulin, which leads to reductions in circulating estrogen and androgens. The aforementioned findings suggest that metformin serves an important role in the treatment of EC. Increased understanding of the mechanism of metformin in EC may provide novel insights into the treatment of this malignancy.
\end{abstract}

\section{Contents}

1. Introduction

2. Metformin indirectly inhibits the development of EC

3. Metformin directly inhibits the development of EC

Correspondence to: Professor Jianqing Hou or Dr Ying Yang, Department of Gynecology and Obstetrics, Yantai Yuhuangding Hospital Affiliated to Qingdao University, 20 Yuhuangding East Road, Zhifu, Yantai, Shandong 264000, P.R. China

E-mail: prohoujianqing@outlook.com

E-mail: hxdany2004@126.com

*Contributed equally

Key words: endometrial cancer, metformin, medical treatment, insulin resistance, signaling pathway
4. Clinical studies of the therapeutic effect of metformin on EC

5. Conclusion

\section{Introduction}

Endometrial cancer (EC) is a malignant gynecological disease that is prevalent in the developed world. It has been estimated that $\sim 63,230$ women were diagnosed with EC and 11,350 women succumbed to this malignancy in 2018 in the United States alone (1). The etiology of EC has not yet been clearly elucidated. Traditionally, the 'unopposed estrogen' hypothesis has been considered to explain the carcinogenesis of EC (2). According to this theory, without sufficient progestins to oppose them, excessive endogenous and/or exogenous estrogens stimulate proliferation and inhibit apoptosis of the endometrium. This process has been considered to contribute to the pathogenesis of EC. Data from numerous studies suggest that insulin resistance is associated with a high risk of EC (3). Our previous study revealed that elevated insulin levels, which is associated with insulin resistance, is an independent risk factor for EC in premenopausal women (4). Another study has reported that insulin resistance-induced hyperinsulinemia independently increases the risk of EC in postmenopausal women (5). Furthermore, another of our previous studies demonstrated that hyperinsulinemia is positively associated with lymph node metastasis in patients with EC and indicates a poor prognosis (6).

At present, surgery is the most effective treatment option for this malignant disease. According to the National Comprehensive Cancer Network guidelines, a surgical procedure including total hysterectomy and bilateral accessory resection, pelvic lymph node dissection and para-aortic lymph node dissection is the most effective treatment method for EC (7). However, this surgical procedure leads to a permanent loss of fertility in patients with EC. According to a previous report (8), $25 \%$ of patients with EC are premenopausal, and $3 \sim 5 \%$ of these patients are $<40$ years old and want to retain fertility. Among the latter patients, 70-88\% have not completed childbearing, and most of them exhibit early-stage highly differentiated endometrioid endometrial carcinoma with a good prognosis. Additionally, some patients cannot undergo 
surgery due to serious complications. Currently, progesterone is widely used in the conservative treatment of EC. However, $>30 \%$ of patients are insensitive or resistant to progesterone treatment (9). Furthermore, side effects, including thrombosis, liver damage, weight gain and progesterone resistance, greatly limit the application of this agent (10).

Metformin is a well-known first-line agent for the treatment of type 2 diabetes, which inhibits hepatic glucose output and intestinal glucose absorption, and promotes the uptake of glucose by skeletal muscle to alleviate insulin resistance. Metformin has also been suggested to be a potential anticancer agent (11). Studies have reported that metformin inhibits the proliferation of a variety of tumor cells, including gastric cancer, pancreatic cancer, medullary thyroid carcinoma and EC cells, in a dose-dependent manner (12-15). The mechanism of this inhibitory effect has been suggested to be associated with cell cycle arrest and the promotion of apoptosis (16). Our previous study revealed that the combined application of metformin and progestins synergistically inhibits the proliferation of EC cells, and suggested that downregulation of the expression levels of cyclin D1 and cyclin E may be an underlying mechanism of this synergistic inhibitory effect (17). Based on the aforementioned results, it may be concluded that metformin is a promising therapeutic option for EC. The anticancer effect of metformin in EC appears to be mediated via direct and indirect mechanisms, which are described in the following sections of this review. Relevant articles and studies were identified using Medline with the following key words alone or in combination: 'Endometrial cancer', 'metformin', 'treatment', 'signaling pathway', 'insulin resistance', 'insulin', 'inflammatory factor' and 'adipokines'.

\section{Metformin indirectly inhibits the development of EC}

Association between insulin resistance and EC. Insulin resistance is characterized as a reduction in the insulin sensitivity of body tissues during metabolic activity, leading to an increase in circulating insulin levels (18). Insulin resistance is known to reduce the insulin-mediated utilization of glucose by the body. This has been suggested to be one of the most important mechanisms of the pathogenesis of diabetes (19). Furthermore, it has been noted that conditions other than diabetes that are closely associated with insulin resistance, namely hypertension, coronary heart disease and hyperlipidemia, also serve important roles in the pathogenesis of certain malignant diseases such as EC, breast cancer and colon cancer (20).

In cases of insulin resistance, particularly in the early stages, circulating insulin levels may be higher than normal (21). Insulin, which is produced and secreted by pancreatic $\beta$ cells, regulates glucose homeostasis by regulating hepatic glucose production and the uptake of glucose by fat and muscle tissue (22). The role insulin serves in tissues is mediated via the insulin receptor, which comprises insulin receptor $\alpha(\operatorname{IR} \alpha)$ and IR $\beta$ subtypes. IR $\alpha$ has a ligand-binding domain that is activated by insulin-induced autophosphorylation. Total insulin receptor and IR $\alpha$ expression levels in EC tissues have been identified to be higher than those in normal endometrial tissues in vivo, whereas in vitro, the overexpression of IR $\alpha$ has been shown to be positively associated with EC cell proliferation (23). This suggests that the activation of insulin signaling is likely to be closely associated with the carcinogenesis of EC. Furthermore, insulin has been suggested to act as a direct mitogenic promoter in the malignant transformation of the endometrium (24). Additionally, it has been reported that excessive insulin inhibits the production of sex hormone binding globulin (SHBG), leading to increased levels of free estrogens and androgens (25). Subsequently, excessive estrogens promote the carcinogenesis of EC according to the 'unopposed estrogen' hypothesis, while excessive androgens provide additional substrate for aromatase-catalyzed biotransformation to estrogen in adipose tissue, resulting in increased circulating levels of estrogen that stimulate the pathogenesis of EC.

There is evidence to suggest that diseases associated with insulin resistance are also risk factors of EC. Body mass index (BMI) is an effective indicator of obesity. It has been reported that patients with a higher BMI usually have a higher risk of EC (26). A clinical study found that elevated BMI value means increased EC risk (27). Furthermore, in another study, the waist-to-hip ratio of patients with EC was shown to be markedly higher than that of control patients with benign endometrial lesions (28), which is consistent with previous findings that upper body obesity is closely associated with EC risk (29). Obese patients often have excessive adipose tissue in which estrogen biosynthesis can occur. Additionally, greater amounts of adipose tissue are usually associated with higher levels of adipokines and inflammatory factors, and these have been suggested to serve an important role in the carcinogenesis of EC $(30,31)$. Type 2 diabetes is another risk factor for both insulin resistance and EC. Increased serum insulin levels have been reported to increase the risk of EC in the early stage of diabetes in a dose-dependent manner (32), and hyperinsulinemia is considered to be an independent risk factor for EC (33). In addition to increasing the incidence of $\mathrm{EC}$, type 2 diabetes also increases the risk of death in patients with EC (34). Furthermore, type 2 diabetes combined with obesity markedly increases EC risk (35). The fasting insulin levels and insulin resistance index values of patients with EC have been shown to be higher than those of controls with benign endometrial lesions (28). Insulin resistance is not only a disease, but also a key pathophysiological process in obesity, diabetes, hypertension and metabolic syndrome. Strategies to promote weight loss, including dietary adjustments and regular physical activity, have been suggested to effectively decrease the risk of insulin resistance as well as that of EC (36).

Metformin inhibits the development of EC by ameliorating insulin resistance. Our previous review suggested that insulin resistance serves a central role in the pathogenesis of EC (3). As an effective insulin sensitizer, metformin greatly improves the utilization of insulin by body tissues to ameliorate insulin resistance. As a result, circulating insulin levels are decreased, which decreases the risk of EC induced by excessive insulin (37). Additionally, the downregulation of circulating insulin levels is considered helpful in the control of body weight, and reductions in body weight have been suggested to inhibit the carcinogenesis of EC (38). This may be at least partly explained by downregulated levels of adipokines and inflammatory factors. A clinical trial has demonstrated that 
metformin delays the development of obesity by improving the resistance status of leptin and insulin growth factor-1 decreasing the levels of insulin, which inhibits the development of EC (39). Additionally, metformin has been reported to increase adiponectin gene expression levels in obese patients, promote the secretion of adiponectin from adipose tissue and thereby induce the apoptosis of EC cells (40). Furthermore, the loss of adipose tissue due to body weight reduction results in less tissue being available for aromatase-catalyzed estrogen biosynthesis. Overall, it may be concluded that metformin indirectly inhibits the development of EC by ameliorating insulin resistance.

\section{Metformin directly inhibits the development of EC}

Metformin has been reported to inhibit the proliferation, migration and invasion of EC cells, and to promote tumor cell apoptosis. However, the specific mechanism remains unclear. Current data suggest that metformin may inhibit the development of EC via a number of pathways described hereinafter.

Hepatic kinase B1 (LKB1)-AMP-activated protein kinase (AMPK)-mTOR signaling pathway. Metformin has been reported to increase glucose uptake by activating the AMPK signaling pathway (41). AMPK is a heterotrimeric serine/threonine protein kinase complex composed of a catalytic $\alpha$ subunit and regulatory $\beta$ and $\gamma$ subunits. AMPK has been suggested to be responsible for the regulation of energy metabolism. It is activated by the cellular stress induced by decreased cellular energy levels and an increased AMP/ATP ratio (42). Once activated, AMPK restores cellular energy levels by stimulating catabolic signaling pathways, including glucose uptake, glycolysis and fatty acid oxidation, and inhibiting ATP-depleting processes, such as fatty acid, cholesterol and protein synthesis (43). LKB1, also known as serine/threonine kinase 11, is an upstream kinase of AMPK that is generally considered to be a tumor suppressor gene. Biochemical and genetic analyses have revealed that under energy stress, LKB1 activates AMPK by phosphorylating AMPK (44). Loss of LKB1 gene expression has been identified in $\sim 65 \%$ of patients with EC (45). LKB1 expression is inversely associated with tumor grade and stage; the inactivation or downregulation of LKB1 promotes the progression of $\mathrm{EC}$ and its loss promotes a highly invasive phenotype. As a tumor suppressor gene, LKB1 may inhibit the occurrence and development of EC via the LKB1-AMPK-mTOR signaling pathway (46).

Metformin enters cancer cells via the organic cationic transporter, and inhibits the activity of the respiratory transporter complex, thereby reducing ATP production. The reduction in ATP level activates the tumor suppressor gene LKB1, which then phosphorylates AMP (46). AMPK regulates several signaling pathways and controls cell proliferation, primarily via inhibition of the mTOR signaling pathway. The available data suggest that the antiproliferative effect of metformin on cancer cells is likely to be mediated via the LKB1-AMPK signaling pathway. For example, in a study of breast cancer cells, metformin activated AMPK by phosphorylating LKB1, and thus inhibited tuberous sclerosis complex 2 phosphorylation and mTOR pathway activation, resulting in the proliferation and apoptosis of the cells being decreased and increased, respectively (47). Also, the results of an in vitro study using ECC-1 and Ishikawa cells demonstrated that metformin reduced cell proliferation and increased AMPK activation in a dose-dependent manner, and indicated that the mTOR signaling pathway inhibition contributed to these effects (48). Metformin has been found to be functionally similar to an mTOR inhibitor, and the PTEN signaling pathway, which is tightly associated with the carcinogenesis of EC, may also be a target of metformin (49). Experiments in mice demonstrated a $50 \%$ reduction in the weight of EC xenografts following treatment with metformin, mediated via inhibition of the LKB1-AMPK-mTOR signaling pathway (50). Metformin has also been revealed to suppress the development of EC xenografts and ameliorate metabolism disorders in obese mice, with the LKB1-AMPK-mTOR signaling pathway being suggested to be partly responsible for the therapeutic effects (51).

PI3K-Akt signaling pathway. PI3K is a cytoplasmic enzyme that is an important member of the phospholipase family, promoting cell proliferation (52). It has both lipid and protein kinase activities. According to the composition of the lipid kinase, PI3K is divided into three subclasses: I, II and III. Class I PI3K is a heterodimer consisting of a catalytic subunit and a regulatory subunit. It is divided into two subclasses: IA (composed by PIK3 CA, PIK3 CB and PIK3 CD) and IB (encoded by PIK3 CG). PIK3 CA is more susceptible to mutation than PIK3 CB (53), and has been reported to serve an important role in the development of several malignant diseases (54). Class I PI3K phosphorylates phosphatidylinositol 4,5 diphosphate (PIP2) to form 3,4,5-trisphosphate phosphatidylinositol (PIP3). Subsequently, PIP3 acts as a second intracellular messenger that is involved in several molecular biological events such as the promotion of cell proliferation and the inhibition of apoptosis (55). PTEN is a phosphatase gene homologous to tensin, which is located on human chromosome 10 q23 and acts as a tumor suppressor gene. PTEN dephosphorylates PIP3 to form PIP2, which serves a negative regulatory role in the downstream signaling pathway of PI3K (56). The activation of PI3K and functional inactivation of PTEN by mutation have been identified in several human malignant tumors, suggesting that PI $3 \mathrm{~K}$ is associated with tumor pathogenesis (57). Protein kinase B, also known as Akt, is a downstream signaling molecule of PI3K that is activated by the phosphorylation of phosphatidylinositol family members on the cell membrane. Subsequently, activated Akt phosphorylates the Ser196 of cysteinyl aspartate specific proteinase (caspase)-3 and caspase-9, thereby inhibiting apoptosis. The lipid phosphatase activity of PTEN has a tumor-suppressing effect mediated via specific dephosphorylation of the phosphatidylinositol D3 ring, which antagonizes phosphorylation of the PI3K substrate and negatively regulates the PIK3-Akt signal transduction pathway (58).

PIK3CA mutation and PTEN deletion are common molecular biological events in EC (59). Clinical data have revealed that the upregulation of PI3K expression and downregulation of PTEN expression frequently occur in patients with EC (60). In vitro data have demonstrated that the PI3K-Akt signaling pathway contributes to the carcinogenesis of EC (61). A study 
of patients with EC observed that PTEN expression is negatively associated with myometrial invasion depth, indicating that PTEN may play an important role of in the development of EC and is potentially a prognostic indicator of EC (62). It has been reported that metformin inhibits Akt expression and stimulates PTEN and IP3 expression via inhibition of the PI3K-Akt signaling pathway (63). This is considered to be an important mechanism via which metformin inhibits the development of EC; both in vitro and in vivo evidence suggest that inhibition of the PI3K-Akt signaling pathway is a key mechanism via which metformin suppresses the carcinogenesis and metastasis of EC (64). A study demonstrated that metformin inhibits the proliferation and colony formation of EC cells in a time- and dose-dependent manner, and suggested that the PI3K-Akt signaling pathway is partly responsible for this inhibitory effect (65). Furthermore, endometrial hyperplasia has been shown to be reversed by metformin management, with suppression of the PI3K-Akt-mTOR signaling pathway appearing to serve a key role in this inhibitory effect (37). Additionally, it has been suggested that metformin management is an effective preventative strategy for EC (63).

Insulin-like growth factor 1 (IGF-1)-associated signaling pathway. IGF-1 is an important member of the IGF family, promoting cell proliferation (66). It is a 70-amino-acid peptide that is synthesized by the liver and is structurally similar to insulin. IGF-1 promotes cell division and has anti-apoptotic effects; it promotes tissue cell proliferation, inhibits apoptosis and regulates tumor pathogenesis via autocrine and paracrine mechanisms (67). As a regulator of cell proliferation, IGF-1 accelerates the transport of amino acids and glucose into cells, thereby increasing protein synthesis and reducing protein degradation, and exerts strong mitogenic effects (68). When IGF-1 binds to the IGF-1 receptor (IGF-1R), PI3K-Akt is activated and the IGF-1-PI3K-Akt signaling pathway is stimulated. Furthermore, IGF-1 also activates the mitogen-activated protein kinase-ERK signaling pathway, thereby inducing tumor cell transcription (69). The overexpression of IGF-binding protein-1 (IGFBP-1) has been shown to inhibit the IGF-1 signaling pathway and reduce the uterine response to IGF-1 (23). In the normal endometrium, IGF-1 expression is induced by estrogen; however, IGFBP-1 suppresses IGF-1 expression and its activity (70). In addition, insulin increases the biological activity of IGF-1 by downregulating IGFBP-1 expression in the endometrium (71). It has been reported that EC cells synthesize and secrete IGF-1, which regulates cell proliferation and differentiation via autocrine and paracrine mechanisms, resulting in continuous proliferation of the cells (72).

As an insulin sensitizer, metformin decreases circulating insulin levels and thereby inhibits the IGF-1-induced phosphorylation of Akt (39). Metformin has been found to not only block the regulatory effect of feedback from the IGF-1R signaling pathway, but also to induce tumor cell apoptosis when combined with IGF-1R inhibitors (73). Clinical data suggest that a regular dose (500 $\mathrm{mg} / \mathrm{time}, 3$ times per day) of metformin effectively reduces elevated IGF-1 levels in the circulation of patients with EC and decreases IGF-1 expression in cancer tissues (74). In an in vitro study, metformin delayed and prevented the IGF-1R feedback-induced proliferation of EC cells, with high concentrations of metformin markedly promoting the apoptosis of EC cells (67). In an in vivo study, the intraperitoneal injection of metformin markedly reduced the circulating IGF-1 levels in mice and strongly inhibited the development of xenograft tumors (75). In addition to suppressing the proliferation-promoting effect of IGF-1 and IGF-2, metformin has also been shown to increase progesterone receptor expression, which appears to be beneficial in the treatment of EC (72).

Apoptosis-stimulating effect. Caspases are a group of cytoplasmic proteases with similar structures. They are responsible for selectively cleaving certain proteins, which leads to apoptosis. Genetic polymorphisms in caspase genes may affect the risk of cancer by altering the expression levels and function of these genes (76). Caspase-3 and -7 have been identified as key performers of cell apoptosis and serve a central role in the execution phase of apoptosis (77). Caspase- 8 is required for death receptor-mediated apoptosis, whereas caspase- 9 is required for mitochondria-mediated apoptosis. The activation of caspase- 8 and -9 induces the subsequent activation of other cysteine proteases and promotes cell apoptosis (78). Inactivating mutations of the caspase gene have been suggested to be associated with the pathogenesis of certain human solid cancers, including EC (76). Metformin activates caspase-8/9 by promoting the electron transport chain and membrane permeability, and the activation of caspase $8 / 9$ leads to the subsequent activation of other caspase enzymes, thereby reducing cell proliferation and promoting apoptosis $(79,80)$. The stimulating effect of metformin on members of the caspase family is dose-dependent (81).

Autophagy refers to the process of cell degradation induced by exogenous stimuli, in which degradation products, such as those derived from the endoplasmic reticulum and Golgi apparatus are recovered and transported to the lysosome for catabolism in order to recycle energy and maintain the stability of the intracellular environment (82). As a signaling pathway for cell survival, autophagy suppresses the cellular stress response and genomic damage by eliminating abnormally folded proteins and organelles, such as mitochondria and lysosomes, from tumor cells, thereby suppressing the occurrence of cancer. The expression levels of Beclin 1, an autophagy-associated gene, have been shown to be positively associated with the tumor grade and degree of myometrial infiltration in EC (83). High Beclin expression is associated with high expression of hypoxia inducible factor $1 \alpha$. Increased autophagy appears to aid tumor survival in a hypoxic microenvironment (84). Microtubule-associated protein 1A/1B-light chain 3 (LC3) expression may be considered a marker of excessive autophagy in EC (85). It has been reported that metformin induces autophagy in colon cancer, melanoma and Ishikawa EC cells (86). Upregulation of the expression levels of autophagy-associated genes, including LC3 and Beclin 1, is considered to be a mechanism via which metformin promotes cell autophagy (87). Furthermore, the knockdown of Beclin 1 expression or inhibition of caspase-3/7 has been shown to inhibit metformin-induced cell autophagy and promote cell proliferation (88). Therefore, metformin promoted autophagy, induced apoptosis and suppressed cell survival in ovarian cancer cells (89). 


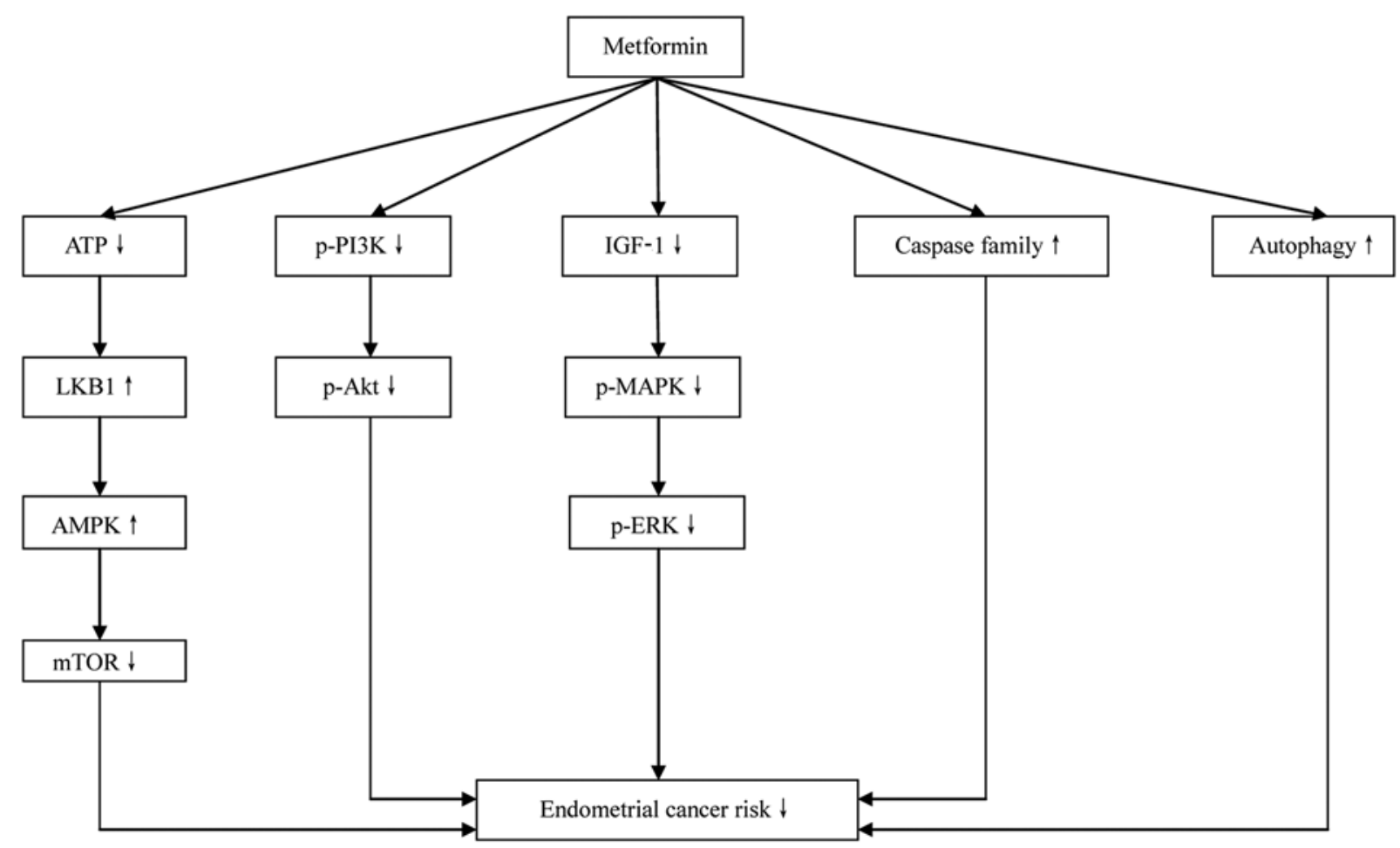

Figure 1. Mechanisms by which metformin directly inhibits the development of endometrial cancer. LKB1, hepatic kinase B1; AMPK, AMP-activated protein kinase; p, phospho; IGF-1, insulin-like growth factor 1; MAPK, mitogen-activated protein kinase.

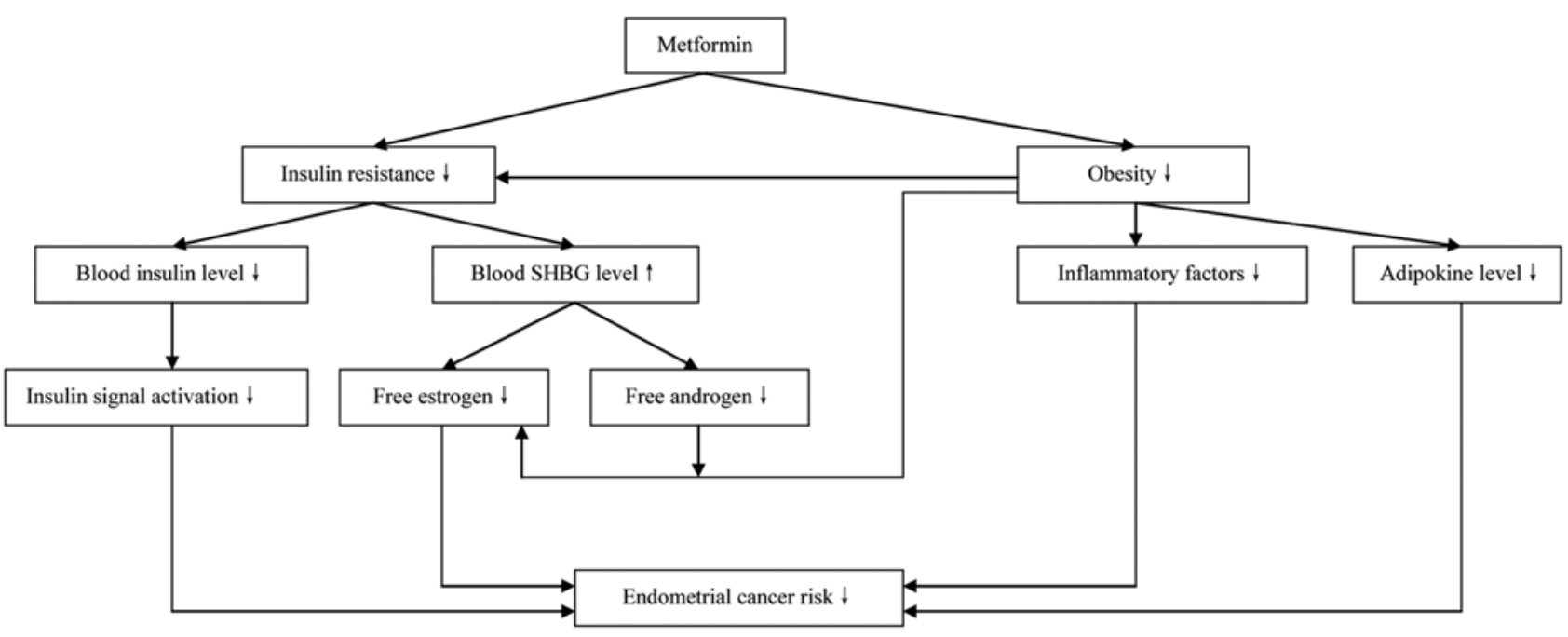

Figure 2. Mechanisms by which metformin indirectly inhibits the development of endometrial cancer. SHGB, sex hormone globulin binding.

\section{Clinical studies of the therapeutic effect of metformin on EC}

Unfortunately, clinical data regarding the effect of metformin in EC are limited. The results provided by different studies are conflicting. One study revealed that the use of metformin in women with diabetes is associated with a lower overall risk of EC (90). However, another study indicated that metformin does not significantly affect the risk of EC (91). Although some studies have investigated the association between metformin use and the survival of patients with EC, whether the use of metformin is associated with improved clinical outcomes in patients with EC remains unclear. In one study, it was demonstrated that patients who did not receive metformin had a 2.3-fold increased risk of mortality compared with patients receiving metformin after adjustments for age, stage, grade, histology and adjuvant treatment (92). However, another study revealed that the overall survival of patients with EC who had or had not received metformin treatment was similar after adjusting for confounding covariates (93). Endometrial hyperplasia is an important precancerous condition of EC. Clinically, progesterone agents and levonorgestrel intrauterine devices have been widely used in the treatment of endometrial hyperplasia. However, the side effects and continued 
risk of recurrence associated with these therapeutic methods have been reported in several studies (94). In a small-scale clinical study, metformin was used in combination with medroxyprogesterone acetate in the treatment of endometrial hyperplasia and EC limited to the endometrium. After 36 weeks, 29 out of 36 patients achieved a complete response. It was noted that 6 of 36 patients suffered diarrhea and nausea when the daily dose of metformin was $2,250 \mathrm{mg}$ (95). However, it was not confirmed whether there were any other potential causes of these side effects. As a novel medication in the treatment of endometrial hyperplasia and EC, the effect of metformin requires further investigation.

\section{Conclusion}

In summary, in vivo and in vitro evidence suggests that metformin serves an important role in the treatment of EC. Overall, metformin directly (Fig. 1) and indirectly (Fig. 2) inhibits the development of EC. The direct mechanism involves inhibition of the LKB1-AMPK-mTOR, PI3K-Akt signaling pathways. Furthermore, IGF-1-associated signaling pathways are inhibited by metformin. Metformin also inhibits the development of EC through its effect on caspase family members and the stimulation of autophagy. Insulin resistance is an established EC risk factor that is important in the indirect mechanism of metformin. The risk factors for insulin resistance also promote the carcinogenesis of EC. In the state of insulin resistance, decreased insulin sensitivity of the body tissues results in elevated levels of circulating insulin. Subsequently, excessive insulin downregulates SHBG levels and upregulates estrogen and androgen levels in the blood, which stimulates the pathogenesis of EC. As an insulin sensitizer, metformin effectively promotes the utilization of insulin by the body tissues, which reduces circulating insulin levels and thereby decreases the insulin-associated risk of EC development.

In conclusion, although the experimental data support the therapeutic effect of metformin in EC, the detailed mechanisms of the therapeutic effect of metformin remain unclear. Furthermore, the clinical applicability of metformin alone or in combination with other medications remains uncertain. These topics warrant further investigation in the future.

\section{Acknowledgements}

Not applicable.

\section{Funding}

No funding was received.

\section{Availability of data and materials}

Not applicable.

\section{Authors' contributions}

JH and YY designed and arranged the manuscript. NM, TX and MG wrote the article. MD, QT, LH, GW, ZL and WW found and analyzed the references in Medline, participated in writing the current paper and gave final approval of the version to be published. NM and TX revised the paper. All authors read and approved the final manuscript.

\section{Ethics approval and consent to participate}

Not applicable.

\section{Patient consent for publication}

Not applicable.

\section{Competing interests}

The authors declare that they have no competing interests.

\section{References}

1. Siegel RL, Miller KD and Jemal A: Cancer statistics, 2018. CA Cancer J Clin 68: 7-30, 2018.

2. van Weelden WJ, Massuger LFAG, ENITEC, Pijnenborg JMA and Romano A: Anti-estrogen treatment in endometrial cancer: A systematic review. Front Oncol 9: 359, 2019.

3. Mu N, Zhu Y, Wang Y, Zhang H and Xue F: Insulin resistance: A significant risk factor of endometrial cancer. Gynecol Oncol 125: 751-757, 2012.

4. Shao Y, Cheng S, Hou J, Zuo Y, Zheng W, Xia M and Mu N: Insulin is an important risk factor of endometrial cancer among premenopausal women: A case-control study in China. Tumour Biol 37: 4721-4726, 2016.

5. Arthur RS, Kabat GC, Kim MY, Wild RA, Shadyab AH, Wactawski-Wende J, Ho GYF, Reeves KW, Kuller LH, Luo J, et al: Metabolic syndrome and risk of endometrial cancer in postmenopausal women: A prospective study. Cancer Causes Control 30: 355-363, 2019.

6. Mu N, Dong M, Liu C, Wang X, Cong J, Wang L, Wang X, Lakhani I, Liu X, Hou J, et al: Association between preoperative serum insulin levels and lymph node metastasis in endometrial cancer-a prospective cohort study. Cancer Med 7: 1519-1527, 2018.

7. Amant F, Moerman P, Neven P, Timmerman D, Van Limbergen E and Vergote I: Endometrial cancer. Lancet 366: 491-505, 2005.

8. Carneiro MM, Lamaita RM, Ferreira MC and Silva-Filho AL: Fertility-preservation in endometrial cancer: Is it safe? Review of the literature. JBRA Assist Reprod 20: 232-239, 2016.

9. Koopman E, Blok LJ, Brinkmann AO, Helmerhorst TJ and Huikeshoven FJ: Differential gene expression in progesterone-sensitive and progesterone-insensitive endometrial carcinoma cells. Eur J Obstet Gynecol Reprod Biol 82: 135-138, 1999.

10. Marjoribanks J, Farquhar C, Roberts H, Lethaby A and Lee J: Long-term hormone therapy for perimenopausal and postmenopausal women. Cochrane Database Syst Rev 1: CD004143, 2017.

11. Mallik R and Chowdhury TA: Metformin in cancer. Diabetes Res Clin Pract 143: 409-419, 2018.

12. Gadducci A, Biglia N, Tana R, Cosio S and Gallo M: Metformin use and gynecological cancers: A novel treatment option emerging from drug repositioning. Crit Rev Oncol Hematol 105: 73-83, 2016.

13. Yamana H, Kato K, Kobara H, Fujihara S, Fujita K, Namima D, Fujita N, Kobayashi K, Kamada H, Morishita A, et al: Metformin inhibits proliferation and tumor growth of QGP-1 pancreatic neuroendocrine tumor cells by inducing cell cycle arrest and apoptosis. Anticancer Res 40: 121-132, 2020.

14. Klubo-Gwiezdzinska J, Jensen K, Costello J, Patel A, Hoperia V, Bauer A, Burman KD, Wartofsky L and Vasko V: Metformin inhibits growth and decreases resistance to anoikis in medullary thyroid cancer cells. Endocr Relat Cancer 19: 447-456, 2012.

15. Liu S, Yue C, Chen H, Chen Y and Li G: Metformin promotes Beclin1-Dependent autophagy to inhibit the progression of gastric cancer. Onco Targets Ther 13: 4445-4455, 2020.

16. Ye J, Qi L, Chen K, Li R, Song S, Zhou C and Zhai W: Metformin induces TPC-1 cell apoptosis through endoplasmic reticulum stress-associated pathways in vitro and in vivo. Int J Oncol 55: 331-339, 2019. 
17. Mu N, Dong M, Li L, Xia M, Qv L, Wang Y, Dong C, Chen Y, Zuo Y, Hou J and Xue F: Synergistic effect of metformin and medroxyprogesterone 17acetate on the development of endometrial cancer. Oncol Rep 39: 2015-2021, 2018.

18. Hari Kumar KVS: The good, the bad, and the ugly facets of insulin resistance. Med J Armed Forces India 76: 4-7, 2020.

19. Luc K, Schramm-Luc A, Guzik TJ and Mikolajczyk TP: Oxidative stress and inflammatory markers in prediabetes and diabetes. J Physiol Pharmacol 70: 809-824, 2019.

20. Patel TP, Rawal K, Bagchi AK, Akolkar G, Bernardes N Dias DDS, Gupta S and Singal PK: Insulin resistance: An additional risk factor in the pathogenesis of cardiovascular disease in type 2 diabetes. Heart Fail Rev 21: 11-23, 2016.

21. Yang C, Qu H, Zhao X, Hu Y, Xiong J, Jiang X, Chen Y and Li Z: Plasma secretagogin is increased in individuals with glucose dysregulation. Exp Clin Endocrinol Diabetes: Sep 23, 2019, (Epub ahead of print). doi: 10.1055/a-1001-2244.

22. Kheirandish M, Mahboobi H, Yazdanparast M, Kamal W and Kamal MA: Anti-cancer effects of metformin: Recent evidences for its role in prevention and treatment of cancer. Curr Drug Metab 19: 793-797, 2018.

23. Pollak M: The insulin and insulin-like growth factor receptor family in neoplasia: An update. Nat Rev Cancer 12: 159-169, 2012.

24. Eritja N, Mirantes C, Llobet D, Yeramian A, Bergadà L, Dosil MA, Domingo M, Matias-Guiu X and Dolcet X: Long-term estradiol exposure is a direct mitogen for insulin/EGF-primed endometrial cells and drives PTEN loss-induced hyperplasic growth. Am J Pathol 183: 277-287, 2013.

25. Arcidiacono B, Iiritano S, Nocera A, Possidente K, Nevolo MT, Ventura V, Foti D, Chiefari E and Brunetti A: Insulin resistance and cancer risk: An overview of the pathogenetic mechanisms. Exp Diabetes Res 2012: 789174, 2012.

26. Kliemann N, Murphy N, Viallon V, Freisling H, Tsilidis KK, Rinaldi S, Mancini FR, Fagherazzi G, Boutron-Ruault MC, Boeing $\mathrm{H}$, et al: Predicted basal metabolic rate and cancer risk in the European prospective investigation into cancer and nutrition. Int J Cancer 147: 648-661, 2020.

27. Bjorge T, Haggstrom C, Ghaderi S, Nagel G, Manjer J, Tretli S, Ulmer H, Harlid S, Rosendahl AH, Lang A, et al: BMI and weight changes and risk of obesity-related cancers: A pooled European cohort study. Int J Epidemiol 48: 1872-1885, 2019.

28. Lai Y and Sun C: Association of abnormal glucose metabolism and insulin resistance in patients with atypical and typical endometrial cancer. Oncol Lett 15: 2173-2178, 2018.

29. Xu WH, Matthews CE, Xiang YB, Zheng W, Ruan ZX Cheng JR, Gao YT and Shu XO: Effect of adiposity and fat distribution on endometrial cancer risk in Shanghai women. Am J Epidemiol 161: 939-947, 2005.

30. Yoon YS, Kwon AR, Lee YK and Oh SW: Circulating adipokines and risk of obesity related cancers: A systematic review and meta-analysis. Obes Res Clin Pract 13: 329-339, 2019.

31. Che Q, Xiao X, Liu M, Lu Y, Dong X and Liu S: IL-6 promotes endometrial cancer cells invasion and migration through signal transducers and activators of transcription 3 signaling pathway. Pathol Res Pract 215: 152392, 2019.

32. Wang Y, Hua S, Tian W, Zhang L, Zhao J, Zhang H, Zhang W and Xue F: Mitogenic and anti-apoptotic effects of insulin in endometrial cancer are phosphatidylinositol 3-kinase/Akt dependent. Gynecol Oncol 125: 734-741, 2012.

33. Gunter MJ, Hoover DR, Yu H, Wassertheil-Smoller S, Manson JE, Li J, Harris TG, Rohan TE, Xue X, Ho GY, et al: A prospective evaluation of insulin and insulin-like growth factor-I as risk factors for endometrial cancer. Cancer Epidemiol Biomarkers Prev 17: 921-929, 2008

34. Anastasi E, Filardi T, Tartaglione S, Lenzi A, Angeloni A and Morano S: Linking type 2 diabetes and gynecological cancer: An introductory overview. Clin Chem Lab Med 56: 1413-1425, 2018.

35. Yang $X$ and Wang J: The role of metabolic syndrome in endometrial cancer: A review. Front Oncol 9: 744, 2019

36. MacKintosh ML, Derbyshire AE, McVey RJ, Bolton J, Nickkho-Amiry M, Higgins CL, Kamieniorz M, Pemberton PW, Kirmani BH, Ahmed B, et al: The impact of obesity and bariatric surgery on circulating and tissue biomarkers of endometrial cancer risk. Int J Cancer 144: 641-650, 2019.

37. Mitsuhashi A, Kiyokawa T, Sato Y and Shozu M: Effects of metformin on endometrial cancer cell growth in vivo: A preoperative prospective trial. Cancer 120: 2986-2995, 2014.

38. Zhang K, Luo Y, Dai $\mathrm{H}$ and Deng Z: Effects of bariatric surgery on cancer risk: Evidence from meta-analysis. Obes Surg 30: 1265-1272, 2020.
39. Soliman PT, Zhang Q, Broaddus RR, Westin SN, Iglesias D, Munsell MF, Schmandt R, Yates M, Ramondetta L and Lu KH: Prospective evaluation of the molecular effects of metformin on the endometrium in women with newly diagnosed endometrial cancer: A window of opportunity study. Gynecol Oncol 143: 466-471, 2016

40. Yates MS, Coletta AM, Zhang Q, Schmandt RE, Medepalli M, Nebgen D, Soletsky B, Milbourne A, Levy E, Fellman B, et al: Prospective randomized biomarker study of metformin and lifestyle intervention for prevention in obese women at increased risk for endometrial cancer. Cancer Prev Res (Phila) 11: 477-490, 2018.

41. Zhou G, Myers R, Li Y, Chen Y, Shen X, Fenyk-Melody J, Wu M, Ventre J, Doebber T, Fujii N, et al: Role of AMP-activated protein kinase in mechanism of metformin action. J Clin Invest 108: 1167-1174, 2001

42. Vallianou NG, Evangelopoulos A and Kazazis C: Metformin and cancer. Rev Diabet Stud 10: 228-235, 2013.

43. Wang Y, An H, Liu T, Qin C, Sesaki H, Guo S, Radovick S, Hussain M, Maheshwari A, Wondisford FE, et al: Metformin improves mitochondrial respiratory activity through activation of AMPK. Cell Rep 29: 1511-1523 e5, 2019.

44. Jiang S, Wang Y, Luo L, Shi F, Zou J, Lin H, Ying Y, Luo Y, Zhan Z, Liu P, et al: AMP-activated protein kinase regulates cancer cell growth and metabolism via nuclear and mitochondria events. J Cell Mol Med 23: 3951-3961, 2019.

45. Pena CG, Nakada Y, Saatcioglu HD, Aloisio GM, Cuevas I, Zhang S, Miller DS, Lea JS, Wong KK, DeBerardinis RJ, et al: LKB1 loss promotes endometrial cancer progression via CCL2-dependent macrophage recruitment. J Clin Invest 125: 4063-4076, 2015.

46. Saraei P, Asadi I, Kakar MA and Moradi-Kor N: The beneficial effects of metformin on cancer prevention and therapy: A comprehensive review of recent advances. Cancer Manag Res 11: 3295-3313, 2019.

47. El-Sisi AE, Sokar SS, El-Sayad ME, Moussa EA and Salim EI: Anticancer effect of metformin against 2-amino-1-methyl-6-phenylimidazo [4,5-b]pyridine-induced rat mammary carcinogenesis is through AMPK pathway and modulation of oxidative stress markers. Hum Exp Toxicol 38: 703-712, 2019.

48. Cantrell LA, Zhou C, Mendivil A, Malloy KM, Gehrig PA and Bae-Jump VL: Metformin is a potent inhibitor of endometrial cancer cell proliferation-implications for a novel treatment strategy. Gynecol Oncol 116: 92-98, 2010.

49. Pabona JMP, Burnett AF, Brown DM, Quick CM, Simmen FA, Montales MTE, Liu SJ, Rose T, Alhallak I, Siegel ER and Simmen RC: Metformin promotes anti-tumor biomarkers in human endometrial cancer cells. Reprod Sci 27: 267-277, 2020.

50. Iglesias DA, Yates MS, van der Hoeven D, Rodkey TL, Zhang Q, Co NN, Burzawa J, Chigurupati S, Celestino J, Bowser J, et al: Another surprise from Metformin: Novel mechanism of action via K-Ras influences endometrial cancer response to therapy. Mol Cancer Ther 12: 2847-2856, 2013.

51. Guo H, Kong W, Zhang L, Han J, Clark LH, Yin Y, Fang Z, Sun W, Wang J, Gilliam TP, et al: Reversal of obesity-driven aggressiveness of endometrial cancer by metformin. Am J Cancer Res 9: 2170-2193, 2019.

52. Moroi AJ and Watson SP: Impact of the PI3-kinase/Akt pathway on ITAM and hemITAM receptors: Haemostasis, platelet activation and antithrombotic therapy. Biochem Pharmacol 94: 186-194, 2015

53. Martini M, De Santis MC, Braccini L, Gulluni F and Hirsch E: PI3K/AKT signaling pathway and cancer: An updated review. Ann Med 46: 372-383, 2014.

54. Guo S, Loibl S, von Minckwitz G, Darb-Esfahani S, Lederer B and Denkert C: PIK3CA H1047R mutation associated with a lower pathological complete response rate in triple-negative breast cancer patients treated with anthracycline-taxane-based neoadjuvant chemotherapy. Cancer Res Treat 52: 689-696, 2020.

55. Jhanwar-Uniyal M, Wainwright JV, Mohan AL, Tobias ME, Murali R, Gandhi CD and Schmidt MH: Diverse signaling mechanisms of mTOR complexes: mTORC1 and mTORC2 in forming a formidable relationship. Adv Biol Regul 72: 51-62, 2019.

56. Johnston SB and Raines RT: Catalysis by the tumor-suppressor enzymes PTEN and PTEN-L. PLoS One 10: e0116898, 2015

57. Kim SM, Nguyen TT, Ravi A, Kubiniok P, Finicle BT, Jayashankar V, Malacrida L, Hou J, Robertson J, Gao D, et al: PTEN Deficiency and AMPK activation promote nutrient scavenging and anabolism in prostate cancer cells. Cancer Discov 8: $866-883,2018$ 
58. Leitner MG, Hobiger K, Mavrantoni A, Feuer A, Oberwinkler J, Oliver D and Halaszovich CR: A126 in the active site and TI167/168 in the TI loop are essential determinants of the substrate specificity of PTEN. Cell Mol Life Sci 75: 4235-4250, 2018.

59. Salvesen HB, Werner HM and Krakstad C: PI3K pathway in gynecologic malignancies. Am Soc Clin Oncol Educ Book: Jun 31, 2013 (Epub ahead of print). doi: 10.1200/EdBook_AM.2013.33.e218.

60. Mackay HJ, Eisenhauer EA, Kamel-Reid S, Tsao M, Clarke B, Karakasis K, Werner HM, Trovik J, Akslen LA, Salvesen HB, et al: Molecular determinants of outcome with mammalian target of rapamycin inhibition in endometrial cancer. Cancer 120: 603-610, 2014.

61. de Melo AC, Paulino E and Garces AH: A review of mTOR pathway inhibitors in gynecologic cancer. Oxid Med Cell Longev 2017: 4809751, 2017.

62. Kosmas K, Mitropoulou G, Provatas I, Stamoulas M and Marouga A: Expression of phosphatase and tensin homologue in imprint smears of endometrial carcinoma. Cytopathology 29: 558-564, 2018.

63. Roncolato F, Lindemann K, Willson ML, Martyn J and Mileshkin L: PI3K/AKT/mTOR inhibitors for advanced or recurrent endometrial cancer. Cochrane Database Syst Rev 10 CD012160, 2019.

64. Saini N and Yang X: Metformin as an anti-cancer agent: Actions and mechanisms targeting cancer stem cells. Acta Biochim Biophys Sin (Shanghai) 50: 133-143, 2018.

65. Qiang P, Shao Y, Sun YP, Zhang J and Chen LJ: Metformin inhibits proliferation and migration of endometrial cancer cells through regulating PI3K/AKT/MDM2 pathway. Eur Rev Med Pharmacol Sci 23: 1778-1785, 2019.

66. Kasprzak A, Kwasniewski W, Adamek A and GozdzickaJozefiak A: Insulin-like growth factor (IGF) axis in cancerogenesis. Mutat Res Rev Mutat Res 772: 78-104, 2017.

67. Zhang Y, Li MX, Wang H, Zeng Z and Li XM: Metformin down-regulates endometrial carcinoma cell secretion of IGF-1 and expression of IGF-1R. Asian Pac J Cancer Prev 16: 221-225, 2015.

68. Tandon M, Chen Z, Othman AH and Pratap J: Role of Runx2 in IGF-1R $\beta /$ Akt- and AMPK/Erk-dependent growth, survival and sensitivity towards metformin in breast cancer bone metastasis. Oncogene 35: 4730-4740, 2016.

69. Chen G, Yuan C, Duan F, Liu Y, Zhang J, He Z, Huang H, He C and Wang H: IGF1/MAPK/ERK signaling pathway-mediated programming alterations of adrenal cortex cell proliferation by prenatal caffeine exposure in male offspring rats. Toxicol App Pharmacol 341: 64-76, 2018.

70. Wang CF, Zhang G, Zhao LJ, Qi WJ, Li XP, Wang JL and Wei LH: Overexpression of the insulin receptor isoform A promotes endometrial carcinoma cell growth. PLoS One 8: e69001, 2013.

71. Shehab MA, Biggar K, Singal SS, Nygard K, Shun-Cheng Li S, Jansson T and Gupta MB: Exposure of decidualized HIESC to low oxygen tension and leucine deprivation results in increased IGFBP-1 phosphorylation and reduced IGF-I bioactivity. Mol Cell Endocrinol 452: 1-14, 2017.

72. Xie Y, Wang JL, Ji M, Yuan ZF, Peng Z, Zhang Y, Wen JG and Shi HR: Regulation of insulin-like growth factor signaling by metformin in endometrial cancer cells. Oncol Lett 8: 1993-1999, 2014.

73. Du J, Shi HR, Ren F, Wang JL, Wu QH, Li X and Zhang RT: Inhibition of the IGF signaling pathway reverses cisplatin resistance in ovarian cancer cells. BMC Cancer 17: 851, 2017.

74. Cai D, Sun H, Qi Y, Zhao X, Feng M and Wu X: Insulin-Like growth factor $1 /$ mammalian target of rapamycin and AMP-Activated protein kinase signaling involved in the effects of metformin in the human endometrial cancer. Int J Gynecol Cancer 26: 1667-1672, 2016.

75. Cao C, Zhou JY, Xie SW, Guo XJ, Li GT, Gong YJ, Yang WJ, $\mathrm{Li}$ Z, Zhong RH, Shao HH and Zhu Y: Metformin enhances nomegestrol acetate suppressing growth of endometrial cancer cells and may correlate to downregulating mTOR activity in vitro and in vivo. Int J Mol Sci 20: 3308, 2019.

76. Xu HL, Xu WH, Cai Q, Feng M, Long J, Zheng W, Xiang YB and Shu XO: Polymorphisms and haplotypes in the caspase-3, caspase-7, and caspase- 8 genes and risk for endometrial cancer: A population-based, case-control study in a Chinese population. Cancer Epidemiol Biomarkers Prev 18: 2114-2122, 2009.
77. McComb S, Chan PK, Guinot A, Hartmannsdottir H, Jenni S, Dobay MP, Bourquin JP and Bornhauser BC: Efficient apoptosis requires feedback amplification of upstream apoptotic signals by effector caspase-3 or -7. Sci Adv 5: eaau9433, 2019.

78. Aral K, Aral CA and Kapila Y: The role of caspase- 8 , caspase-9, and apoptosis inducing factor in periodontal disease. J Periodontol 90: 288-294, 2019.

79. Jang JH, Song IH, Sung EG, Lee TJ and Kim JY: Metformin-induced apoptosis facilitates degradation of the cellular caspase 8 (FLICE)-like inhibitory protein through a caspase-dependent pathway in human renal cell carcinoma A498 cells. Oncol Lett 16: 2030-2038, 2018

80. Zhao Y, Luo Q, Mo J, Li J, Ye D, Ao Z, Chen L and Liu J: Metformin in combination with JS-K inhibits growth of renal cell carcinoma cells via reactive oxygen species activation and inducing DNA breaks. J Cancer 11: 3701-3712, 2020.

81. Zhou X, Zhang P, Wang Q, Ji N, Xia S, Ding Y and Wang Q: Metformin ameliorates experimental diabetic periodontitis independently of mammalian target of rapamycin (mTOR) inhibition by reducing NIMA-related kinase 7 (Nek7) expression. J Periodontol 90: 1032-1042, 2019.

82. Guo Y, Yuan J, Yin S, Wang X, Shuai R and Kang J: MAP2K6-FP enhances the sensitiveness of paclitaxel for ovarian cancer via inducing autophagy. Int J Gynecol Cancer 27: 1082-1087, 2017.

83. Giatromanolaki A, Koukourak is MI, Koutsopoulos A, Chloropoulou P, Liberis V and Sivridis E: High Beclin 1 expression defines a poor prognosis in endometrial adenocarcinomas. Gynecol Oncol 123: 147-151, 2011.

84. Bermudez M, Aguilar-Medina M, Lizarraga-Verdugo E, Avendaño-Félix M, Silva-Benítez E, López-Camarillo C and Ramos-Payán R: LncRNAs as regulators of autophagy and drug resistance in colorectal cancer. Front Oncol 9: 1008, 2019.

85. Ran X, Zhou P and Zhang K: Autophagy plays an important role in stemness mediation and the novel dual function of EIG121 in both autophagy and stemness regulation of endometrial carcinoma JEC cells. Int J Oncol 51: 644-656, 2017.

86. Tomic T, Botton T, Cerezo M, Robert G, Luciano F, Puissant A, Gounon P, Allegra M, Bertolotto C, Bereder JM, et al: Metformin inhibits melanoma development through autophagy and apoptosis mechanisms. Cell Death Dis 2: e199, 2011.

87. Zhang D, Xuan J, Zheng BB, Zhou YL, Lin Y, Wu YS, Zhou YF, Huang YX, Wang Q, Shen LY, et al: Metformin improves functional recovery after spinal cord injury via autophagy flux stimulation. Mol Neurobiol 54: 3327-3341, 2017.

88. Takahashi A, Kimura F, Yamanaka A, Takebayashi A, Kita N, Takahashi K and Murakami T: Metformin impairs growth of endometrial cancer cells via cell cycle arrest and concomitant autophagy and apoptosis. Cancer Cell Int 14: 53, 2014.

89. Zou G, Bai J, Li D and Chen Y: Effect of metformin on the proliferation, apoptosis, invasion and autophagy of ovarian cancer cells. Exp Ther Med 18: 2086-2094, 2019.

90. Tseng $\mathrm{CH}$ : Metformin and endometrial cancer risk in Chinese women with type 2 diabetes mellitus in Taiwan. Gynecol Oncol 138: 147-153,2015.

91. Franchi M, Asciutto R, Nicotra F, Merlino L, La Vecchia C, Corrao $\mathrm{G}$ and Bosetti C: Metformin, other antidiabetic drugs, and endometrial cancer risk: A nested case-control study within Italian healthcare utilization databases. Eur J Cancer Prev 26: 225-231, 2017.

92. Ko EM, Walter P, Jackson A, Clark L, Franasiak J, Bolac C, Havrilesky LJ, Secord AA, Moore DT, Gehrig PA and Bae-Jump V: Metformin is associated with improved survival in endometrial cancer. Gynecol Oncol 132: 438-442, 2014.

93. Rossi A, Garber CE, Ortiz M, Shankar V, Goldberg GL and Nevadunsky NS: Feasibility of a physical activity intervention for obese, socioculturally diverse endometrial cancer survivors. Gynecol Oncol 142: 304-310, 2016.

94. Clement NS, Oliver TR, Shiwani H, Sanner JR, Mulvaney CA and Atiomo W: Metformin for endometrial hyperplasia. Cochrane Database Syst Rev 10: CD012214, 2017

95. Mitsuhashi A, Sato Y, Kiyokawa T, Koshizaka M, Hanaoka H and Shozu M: Phase II study of medroxyprogesterone acetate plus metformin as a fertility-sparing treatment for atypical endometrial hyperplasia and endometrial cancer. Ann Oncol 27: $262-266,2016$ 